\title{
Hox11 paralogous genes are essential for metanephric kidney induction
}

\author{
Deneen M. Wellik, Patrick J. Hawkes, and Mario R. Capecchi ${ }^{1}$ \\ Department of Human Genetics and Howard Hughes Medical Institute, University of Utah School of Medicine, \\ Salt Lake City, Utah 84112-5331, USA
}

\begin{abstract}
The mammalian Hox complex is divided into four linkage groups containing 13 sets of paralogous genes. These paralogous genes have retained functional redundancy during evolution. For this reason, loss of only one or two Hox genes within a paralogous group often results in incompletely penetrant phenotypes which are difficult to interpret by molecular analysis. For example, mice individually mutant for Hoxa11 or Hoxd11 show no discernible kidney abnormalities. Hoxa11/Hoxd11 double mutants, however, demonstrate hypoplasia of the kidneys. As described in this study, removal of the last Hox11 paralogous member, Hoxc11, results in the complete loss of metanephric kidney induction. In these triple mutants, the metanephric blastema condenses, and expression of early patterning genes, Pax2 and Wt1, is unperturbed. Eya1 expression is also intact. Six2 expression, however, is absent, as is expression of the inducing growth factor, Gdnf. In the absence of $G d n f$, ureteric bud formation is not initiated. Molecular analysis of this phenotype demonstrates that Hox11 control of early metanephric induction is accomplished by the interaction of Hox11 genes with the pax-eya-six regulatory cascade, a pathway that may be used by Hox genes more generally for the induction of multiple structures along the anteroposterior axis.
\end{abstract}

[Key Words: Hox; kidney; pax-eya-six; metanephric induction]

Received March 19, 2002; revised version accepted April 15, 2002.

Hox genes have long been recognized as important regulators of embryonic development. In mammals, this complex of 39 genes resides on four separate chromosomal linkage groups, designated A, B, C, and D. These groups diverged early in the evolution of vertebrates following quadruplication of a single ancestral complex. Homologous members within the separate linkage groups are divided into 13 sets of paralogous genes, each having two to four members. During development, paralogous sets of genes are activated sequentially in the developing embryo, with the Hox 1 and Hox2 paralogous groups being expressed earlier and more anteriorly in the embryo and successive groups through paralogous group Hox13 appearing later with increasingly more posterior limits of expression (Duboule and Dolle 1989; Graham et al. 1989). Despite the recognition of the importance of Hox genes in development and extensive genetic analysis of both loss-of-function and gain-of-function mutations in mouse, few hints have surfaced regarding how these genes function at the molecular level to provide positional information along the anteroposterior (A-P) axis.

It has been clear for some time that functional redundancy may account for the difficulty in determining the

${ }^{1}$ Corresponding author.

E-MAIL mario.capecchi@genetics.utah.edu; FAX (801) 585-3425.

Article and publication are at http://www.genesdev.org/cgi/doi/10.1101/ gad.993302. molecular pathways in which Hox genes operate. Lossof-function of two or more paralogous members has repeatedly demonstrated exacerbations of phenotype compared to the single mutants (Condie and Capecchi 1994; Davis et al. 1995; Horan et al. 1995; Zakany et al. 1997; Studer et al. 1998; Chen and Capecchi 1999; Rossel and Capecchi 1999). These studies have pointed out that many of the most basic functions of Hox genes may be conserved among all members of any given paralogous group. Therefore, partial loss-of-function of the paralogous group would be expected to result in what classical geneticists refer to as 'hypomorphic' phenotypes. Although hypomorphic phenotypes are often informative with regards to temporal and spatial aspects of function, they are not characteristically amenable to molecular analysis, particularly without comparison to the complete loss-of-function phenotype.

A corollary to this hypothesis with respect to Hox analysis is that only in the functional absence of an entire paralogous group can the most basic functions of this set of genes be realized. An example is the Hox11 paralogous group genes. Hoxa11 and Hoxd11 single mutant phenotypes have been reported as well as the double mutant phenotype (Small and Potter 1993; Davis and Capecchi 1994; Davis et al. 1995). These genes affect the development of the reproductive tract, the vertebral skeleton, the limb, and the kidney. The severity of all four aspects of phenotype increases in the Hoxa11/ 
Hoxd11 double mutant compared to either single mutant. With respect to kidney development, neither Hoxa11 nor Hoxd11 single mutant animals display any overt phenotype. Double mutant mice, however, demonstrate variably penetrant kidney hypoplasia (Davis et al. 1995; Patterson et al. 2001). In these double mutants, there is one remaining functional Hox11 gene, Hoxc11. If members of the Hox11 paralogous group are functionally redundant in kidney development, then removal of the final member, Hoxc11, should further exacerbate the kidney phenotype and represent the complete loss-offunction phenotype for this paralogous gene family.

The kidney is an excellent model organ system for the study of inductive mechanisms in vivo and in vitro. Early molecular events of kidney morphogenesis are functionally well described (Dressler 1999; Kuure et al. 2000), making it an ideal system in which to analyze Hox function. The metanephric kidney develops through a series of reciprocal inductions. Initially, a caudal region of the intermediate mesoderm termed the metanephric blastema condenses. From its earliest stages, the metanephric blastema expresses each of the three Hox11 paralogs, Hoxa11, Hoxc11, and Hoxd11 (Hsieh-Li et al. 1995; Hostikka and Capecchi 1998; Patterson et al. 2001 and unpubl.). The condensed metanephric blastema induces the ureter to bud from the Wolffian duct. The Hox11 paralogs are never expressed in either the Wolffian duct or the ureteric bud. The ureter, in turn, induces the metanephric mesenchyme to convert to epithelium and form the differentiated components of the nephron. By continued proliferation and branching of the ureter induced by the mesenchyme and continued nephrogenesis in the mesenchyme induced by the ureter, the kidney develops into a mature organ containing thousands of filtration units connected through a complex collecting duct system.

The kidneys in Hoxa11/Hoxd11 double mutants were described in greater detail recently (Patterson et al. 2001). In those embryos, ureteric bud initiation was reported to occur normally at E11.5. By E13.5, the double mutant embryos in that study displayed kidney abnormalities. The ureter had undergone less branching at this stage compared to controls. The expression patterns of many genes, including Wnt7b, Wnt11, Bf2, Pax2, Wt1, and Gdnf were examined at midorganogenesis. Expression of each of the markers was detected in the double mutants, and many were found to be altered compared to controls, particularly with respect to expression along the dorsoventral axis. Since the development of the kidneys at this stage in the double mutants was already morphologically abnormal, it is difficult to discern from this analysis which genes were directly affected by loss of Hox function, and which altered gene expression patterns reflected secondary effects of aberrant kidney morphology.

In the present study, we report the generation of mice

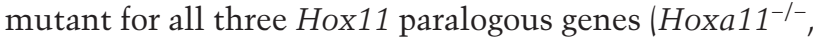
Hoxc11 $1^{-/}$, Hox $111^{-/-}$) and the resulting kidney phenotype. In triple mutants, metanephric induction is completely absent. The early metanephric blastema con- denses, but the ureter never buds from the Wolffian duct. The expression of many early patterning molecules including Pax2, Wt1, and Eya1 is normal. Six2 and Gdnf expression, however, are specifically absent. The molecular analysis of this phenotype appears identical to that reported for Eya1 homozygous mutants and supports the hypothesis asserted by Xu et al. (1999) that the pax-eya-six pathway, first elucidated in Drosophila eye development, is conserved in mammalian kidney development. This study further demonstrates that the Hox11 paralogous genes converge on this pathway during metanephric kidney development.

\section{Results}

Mice with combinations of null mutations for Hoxa11, Hoxc11, and Hoxd11 were generated by standard genetic crosses (Davis and Capecchi 1994; Boulet et al., in prep.; S.L. Hostikka and M.R Capecchi, in prep.). Generation of triple heterozygous animals $(11 \mathrm{AaCcDd}$, where uppercase letters denote wild-type alleles and lowercase letters denote mutant alleles) as well as animals with combinations of four mutant alleles (11aaCcDd, 11AaccDd, or $11 \mathrm{AaCcdd}$ ) were obtained in near Mendelian ratios. All male and female mice of the above genotypes are infertile and show many of the reproductive defects previously reported for Hoxa11 mutant mice (Small and Potter 1993; Hsieh-Li et al. 1995; data not shown). To overcome the problem with infertility, triple mutant embryos were produced by in vitro fertilization (Sztein et al. 1997).

Hoxa11/Hoxc11/Hoxd11 triple mutants have no kidneys

At birth, Hoxa11/Hoxd11 double mutants displayed kidney hypoplasia as described previously (Fig. 1, cf. D and A; Davis et al. 1995; Patterson et al. 2001). These previous reports noted that kidneys were occasionally found unilaterally. Of note, all four Hoxa11/Hoxd11 embryos histologically examined at E18.5 in the present study possessed two hypoplastic kidneys. The other doublemutant combinations (11aaccDD and 11AAccdd) demonstrated less severe but similar defects to those seen in Hoxa11/Hoxd11 double mutants (Fig. 1B,C; all eight embryos examined also had two kidneys). Embryos with five mutant alleles (11aaccDd, 11 aaCcdd, or 11 Aaccdd) were more severely affected. Of the five embryos analyzed at this time point, two possessed two very small, rudimentary kidneys, one had only a single kidney remnant (Fig. 1E), and two embryos had no kidney at this stage. The severity of the phenotype did not correlate with the remaining wild-type allele. In addition, as the number of mutant alleles increases, the hypoplastic kidneys were located at increasingly more caudal and ventral locations, consistent with observations of Patterson et al. (2001), suggesting that migration of the organ is disrupted. Triple mutant animals (11aaccdd) exhibited no metanephric kidney formation (Fig. 1F). 


\section{AACCDd}
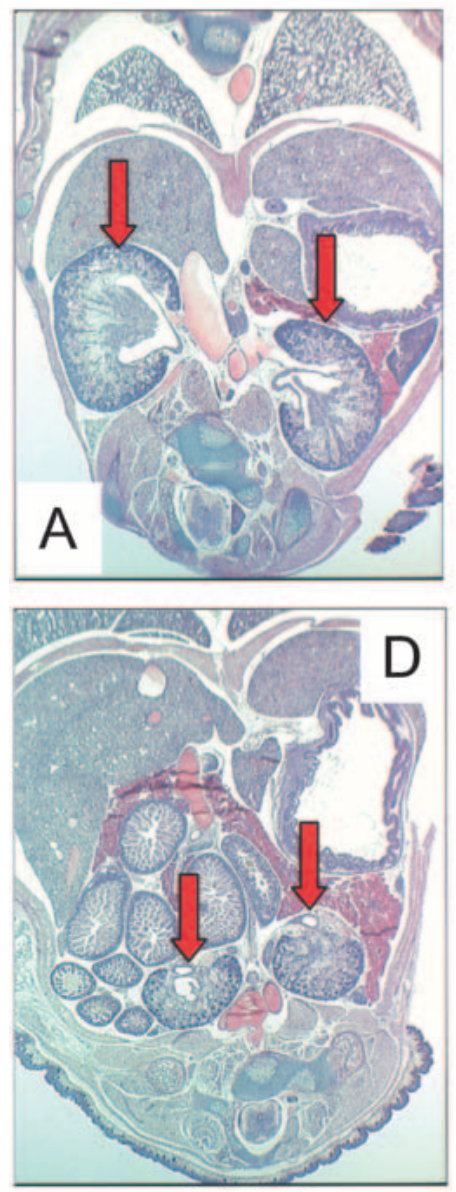

11 aaCCdd
11 AAccdd
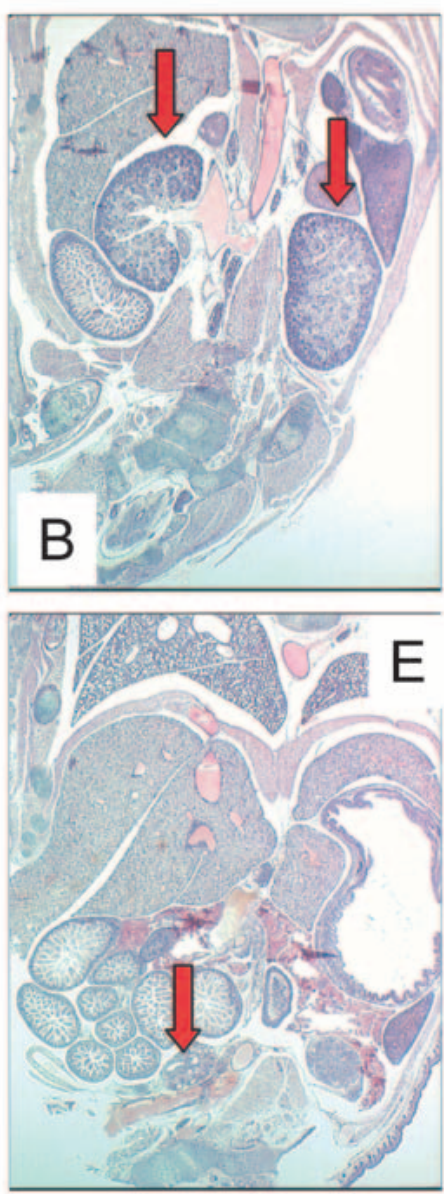

11Aaccdd
11 aaccDD
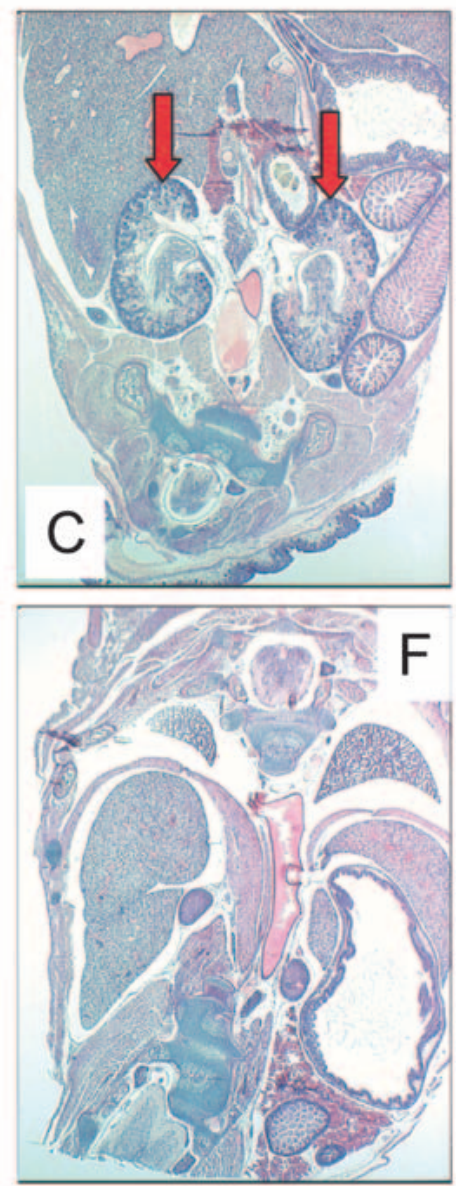

11 aaccdd

Figure 1. Kidney phenotype is exacerbated with increasing Hox 11 mutant allelic contribution. Paraffin-embedded frontal sections 15 $\mu \mathrm{m})$ through E18.5 embryos, stained with hematoxylin and eosin. In all sections, anterior is up. Red block arrows denote kidneys in each panel. Note increasing severity of kidney phenotype with increasing number of mutant alleles $(A-F)$. $(A)$ Wild-type embryo. $(B)$ Double mutant of Hoxc11/Hoxd11. (C) Double mutant of Hoxa11/Hoxc11. (D) Double mutant of Hoxa11/Hoxd11. (E) Five-allele

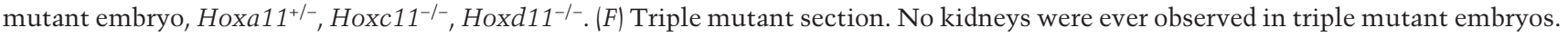

At E13.5 in wild-type embryos, the mesonephros has regressed, the ureter has invaded the metanephric blastema, and a few rounds of branching of the ureter have occurred in the metanephros (Fig. 2C). In the Hoxa11/ Hoxd11 double mutant embryos (and to a lesser extent in all other combinations of four mutant alleles), the ureter's route to the kidney is circuitous and abnormal. It bypasses the mediolateral aspect of the kidney where it normally enters and instead traverses anteriorly around the kidney to the lateral side where it finally enters the metanephric kidney (Fig. 2D). All other fourand five-allele mutant embryos at this stage have two, abnormally developing kidneys (data not shown; $n=6$ ). These findings suggest that the occasional unilateral kidney reported in adult Hoxa11/Hoxd11 double mutant mice and the more frequent unilateral or bilateral absence of metanephric kidney in the five-allele mutant newborns are due to loss of an aberrantly developing structure. Triple mutant embryos at this stage show no sign of metanephric kidney development ( $n=3$; Fig. 2 E).

\section{Ureteric bud induction does not occur in triple mutants}

The initial budding of the ureter from the Wolffian duct was examined in E11.5 embryos. At this stage a single, unbranched ureteric bud can be seen invading the metanephric blastema in control embryos (Fig. 3A). In all mutant embryos examined, condensation of the metanephric blastema appears normal. All Hoxa11/Hoxd11 embryos have a single ureteric bud, and initial bud invasion appears normal for both kidneys in all cases examined $(n=5$, Fig. 3B). In embryos with five mutant alleles, bud invasion can occur, but the ureteric bud examined at the 

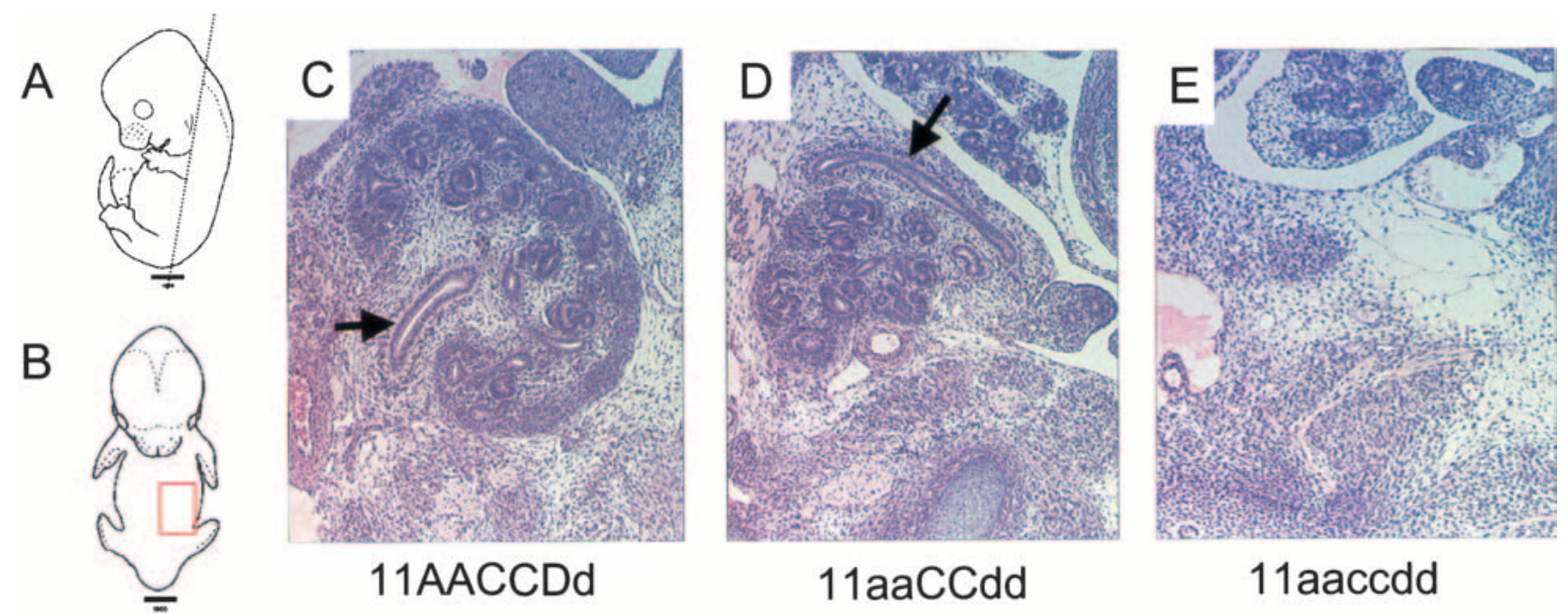

Figure 2. Disrupted ureteric migration at E13.5. Paraffin-embedded histological frontal sections $(5 \mu \mathrm{m})$ through embryos stained with hematoxylin and eosin. Dashed black line in panel $A$ shows the plane of section shown in $C-E$. The red box in panel $B$ shows the approximate region shown in $C-E$. Panels $C-E$ show the embryos' left developing metanephros. The midline of each embryo is to the left, anterior to the top. Black arrows denote the ureter. $(C)$ Control kidney. $(D)$ Section through a Hoxa11/Hoxd11 double mutant embryo. The ureter traverses by the medial aspect of the kidney (not seen in this section), around the anterior aspect of the kidney to the lateral side, where it eventually enters the developing metanephros. No kidney development was seen in triple mutant embryos at this stage $(E)$.

same stage frequently migrated in regions away from the metanephric mesenchyme $(n=7$, Fig. $3 \mathrm{C})$. No ureteric bud formation occurred in any triple mutant embryos examined ( $n=8$, Fig. 3D). As expected in the absence of metanephric induction, the triple mutant blastema was lost due to apoptosis ( $n=4$, Fig. 4A,B; Koseki et al. 1992). Kidney development was not detected beyond this stage in triple mutant embryos.

\section{Molecular analysis of metanephric induction}

Induction of metanephric development is well characterized, and several molecules are known to affect this process. Since each of the Hox11 paralogous genes are expressed in the blastemal mesenchyme and not in the Wolffian duct (Hsieh-Li et al. 1995; Hostikka and Capecchi 1998; Patterson et al. 2001), we analyzed the expression of the mesenchymal molecules known or expected to play a role in blastemal induction of ureteric bud formation. We examined the expression of these genes at E10.5, just prior to initiation of ureteric bud formation. At this stage, all control and mutant embryos are morphologically indistinguishable. The assumption was made that any affect on the Wolffian duct would be secondary to a cell autonomous defect in the blastema where these Hox11 transcription factors are expressed. This analysis was limited to molecules known or expected to have a role in initiating ureteric bud induction. The expression patterns of molecules such as Wnt4 and $B f 2$, which are known to be expressed in the mesenchyme after the initiation of ureteric bud formation and to affect later stages of metanephric development, were not analyzed in this triple mutant study.
Wt1 and Pax2 are transcription factors important for establishing competence and early patterning in kidney development. Mice with targeted disruptions in either of these genes demonstrate loss of metanephric induction (Kreidberg et al. 1993; Torres et al. 1995). Wt1 is expressed in the developing blastema as well as the surrounding developing urogenital mesenchyme, but it is not expressed in the Wolffian duct. Pax2 is expressed in both the metanephric blastema as well as the Wolffian duct. Expression of these two proteins appears normal in Hox11 triple mutant embryos at E10.5, just prior to normal ureteric bud formation (see Fig. 5A,B and C,D, respectively).

Eya1 and Six2 are also expressed in the metanephric blastema at this stage. Loss-of-function of Eya1 in mice results in loss of ureteric bud induction as seen in Hox11 paralogous triple mutants (Xu et al. 1999). Targeted disruption of Six2 has not been reported. In all Hox11 mutant blastemas, Eya1 expression also appears normal (Fig. 5E-G). However, Six2 expression is never detected in the triple mutant blastema (Fig. 5, cf. $\mathrm{H}$ and J). Additionally, the level of Six2 expression appears to be dependent on the number of functional Hox11 alleles, as embryos with five mutant alleles show very weak expression of Six2 compared to controls (Fig. 5H-J).

Expression of $G d n f$ is also absent or greatly reduced in triple mutants compared to controls (Fig. 5K,L). Gdnf is expressed in the metanephric blastema, and Gdnf mutant mice exhibit the same loss-of-induction phenotype as Hox11 triple mutants (Hellmich et al. 1996; Moore et al. 1996; Pichel et al. 1996; Sanchez et al. 1996). Gdnf has been demonstrated to be an inducing ligand in this system, operating through coreceptors c-ret and GDNFR $\alpha$, 

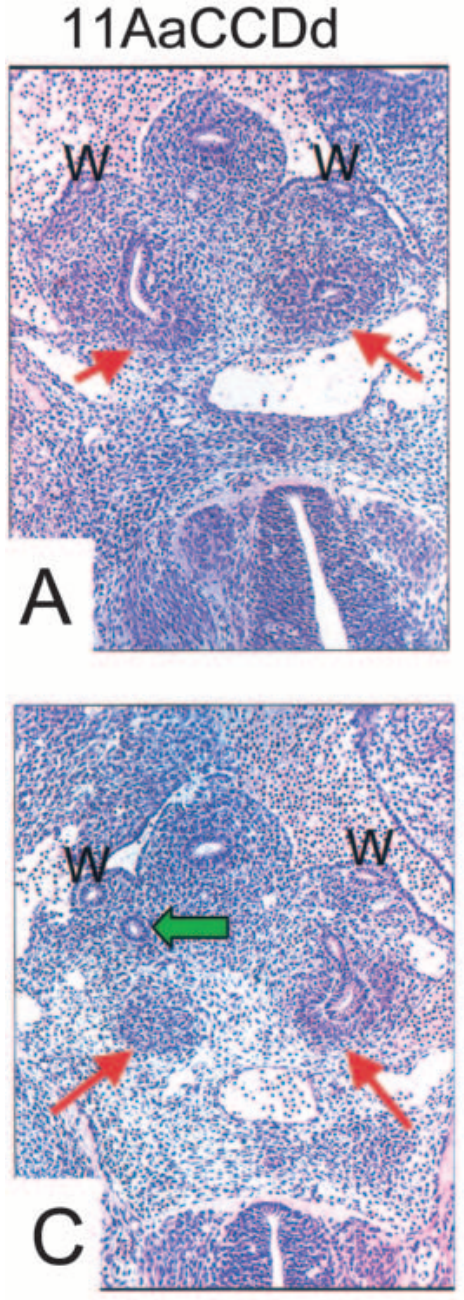

11Aaccdd
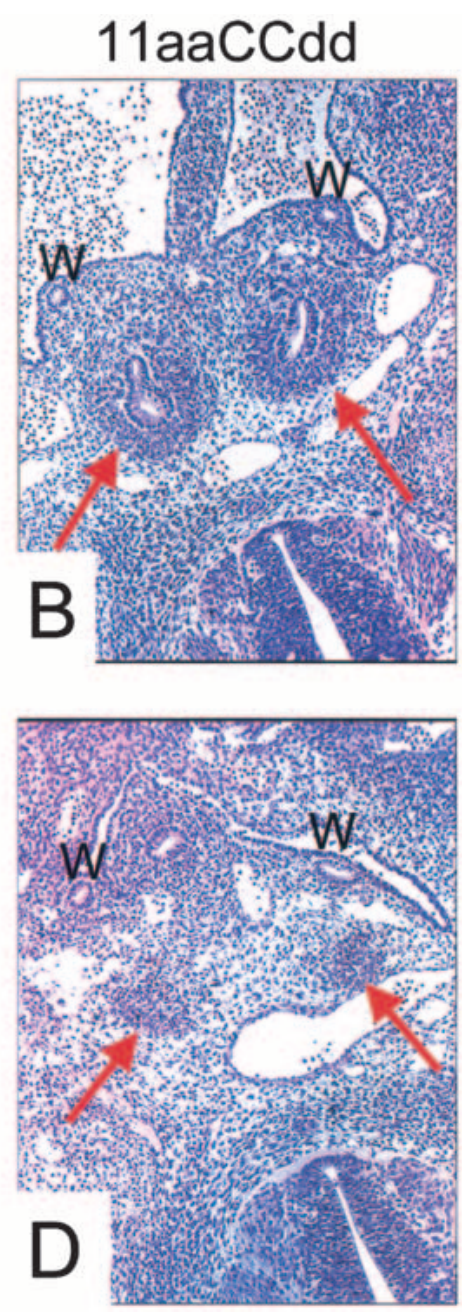

11aaccdd
Figure 3. Ureteric budding is disrupted in Hox11 mutants. Hematoxylin and eosin-stained sections $(5 \mu \mathrm{m})$ of paraffin-embedded E11.5 embryos. W, Wolffian duct. Red arrows denote metanephric blastema. (A) Normal ureteric budding into the blastema of a control embryo. This process appears to occur normally in double mutant embryos (Hoxa11/Hoxd11 double mutant shown in $B)$. The green arrow in panel $C$ shows the right ureteric bud of a five-allele mutant embryo. This bud never makes contact with the metanephric blastema, but can be followed through sections traversing an indirect path around it. $(D)$ A triple mutant embryo. No ureteric bud is ever formed in these embryos. expressed in the Wolffian duct, and Gdnf expression is necessary for ureteric bud induction in vivo and in vitro (Schuchardt et al. 1996; Treanor et al. 1996; Trupp et al. 1996; Sainio et al. 1997; Cacalano et al. 1998).

\section{Discussion}

In this study, we examined the effect of loss-of-function of the entire Hox11 paralogous group on kidney devel-
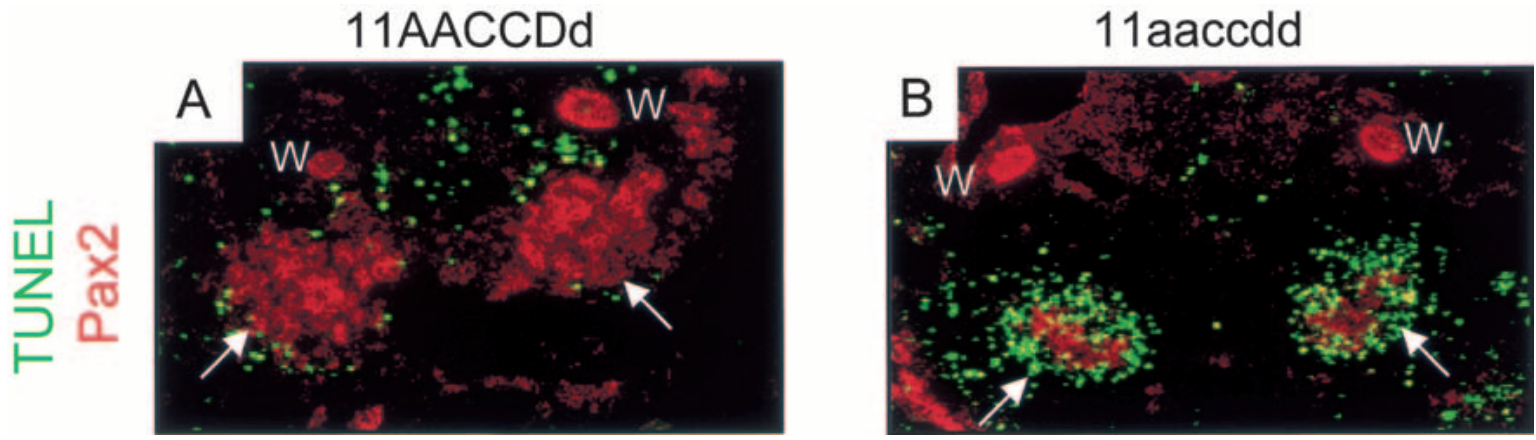

Figure 4. Hox11 triple mutant blastemas undergo apoptosis in the absence of induction. Consistent with loss of metanephric induction, the condensed blastemal mesenchyme is lost by apoptosis in the triple mutant. $(A)$ Control and $(B)$ Hox 11 triple mutant, show sections through E11.5 embryos. Cell death staining by TUNEL is shown in green, and Pax2 antibody staining is in red. Note massive cell death in the triple mutant blastema, but not the Wolffian ducts. The metanephric blastemas in control and mutant embryos are indicated by a white arrow; the Wolffian ducts are indicated by a white W. 
Wellik et al.
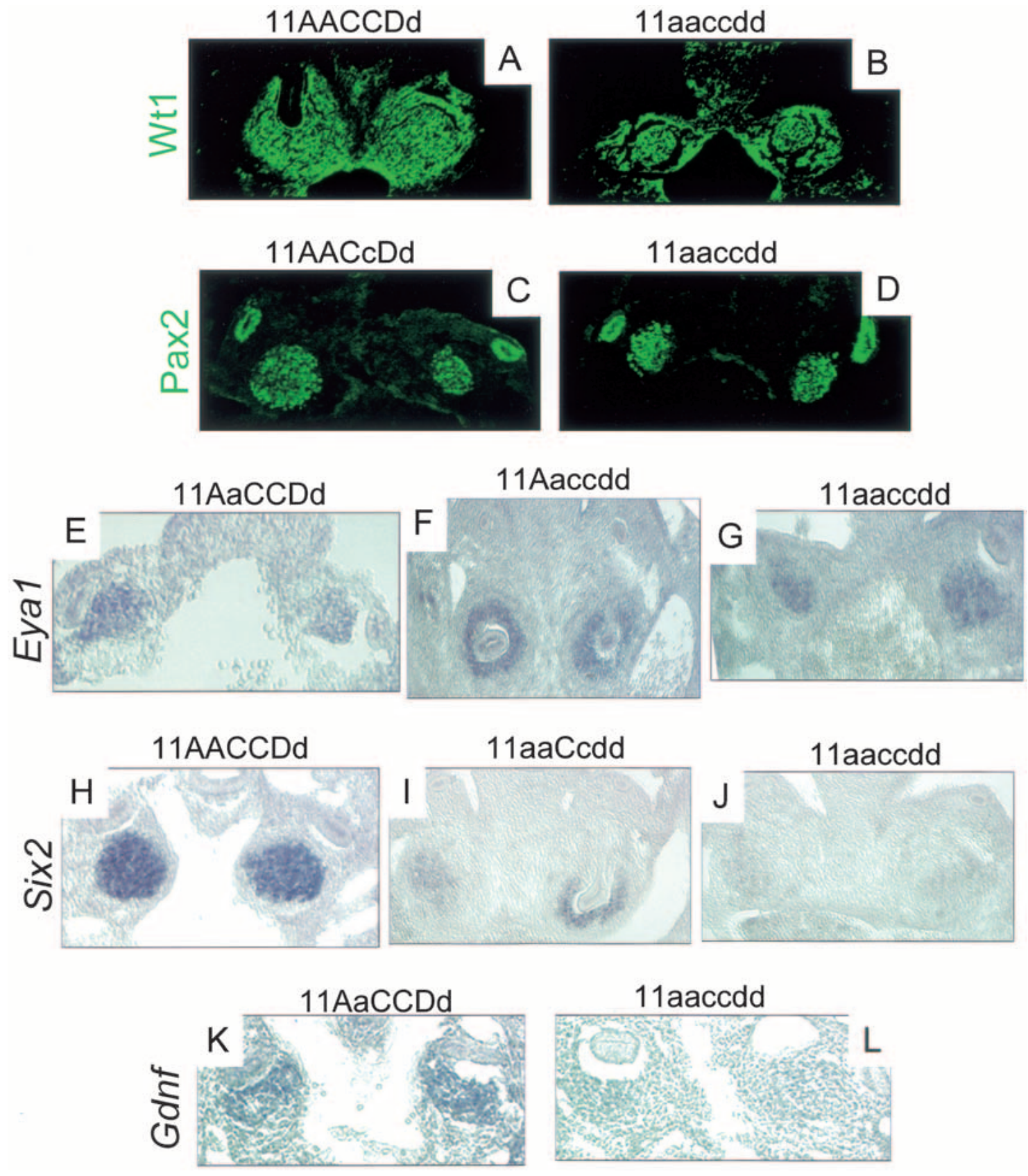

Figure 5. Molecular characterization of Hox11 triple mutant phenotype. $(A-L)$ E10.5 frontal sections through the metanephric region. $(A, B)$ Immunohistochemical staining of $\mathrm{Wt} 1$ in control and triple mutant embryos in the metanephric blastema and surrounding urogenital mesenchyme. Note that the control section in $A$ is at a slightly later stage, approximately E11. At the left side of $A$, the ureter (which does not stain for Wt1) can be seen entering the metanephric mesenchyme. $(C, D) \operatorname{Pax} 2$ immunostaining in the blastema and Wolffian duct. There are no observable differences between control and mutant embryos. (E-G) Eya1 expression as detected by in situ hybridization (ISH). (E) Control embryo. (F) Five-allele mutant, 11Aaccdd, shown at a slightly later stage (approximately E11, note invasion of both blastemas by nonexpressing ureteric bud). (G) Triple homozygous mutant. Expression of Eya1 is normal in mutants and controls. (I) Six2 ISH demonstrates a drastic reduction in Six2 message in five-allele embryos (11aaCcdd shown here at approximately E11, note invasion of the right metanephric blastema by nonexpressing ureteric bud), compared to control expression $(H)$. There is a complete loss of message in triple mutant mesenchyme $(J)$ compared to control $(H)$. Gdnf expression as detected by ISH in control $(K)$ and triple mutant $(L)$ is absent or much reduced in triple mutant blastemas.

opment. The major conclusions are that the Hox11 paralogous genes are functionally redundant in early kidney development and are necessary for the initiation of metanephric development. Removal of all six functional alleles of the Hox11 paralogous group results in a complete loss of ureteric bud formation. Further, while many 
aspects of the early molecular patterning of the metanephric blastema are intact, expression of Six2 and Gdnf was specifically affected. This morphological and molecular phenotype is identical to that reported for Eya1 mutant kidneys (Xu et al. 1999) and suggests that the Hox11 paralogous genes directly impinge on the paxeya-six pathway in kidney development.

\section{Loss of metanephric induction}

In retrospect, it may not seem surprising that disruption of the entire Hox11 paralogous group results in loss of metanephric induction. Hindsight allows us to make this a natural progression, but a more insightful review of the current literature might not suggest this outcome. First, at least 15 of the 39 Hox genes are expressed in the developing kidney (Davies and Brandli 2002). The chance that disruption of three of these 15 Hox genes, albeit an entire paralogous group, would result in loss of initiation of the organ is therefore not intuitive. Secondly, other systems affected by these mutations are not perturbed to this degree. Of the Hox11 paralogs, for instance, Hoxa11 and Hoxd11 are expressed in the developing forelimb zeugopod, whereas all three paralogous members are expressed in the hindlimb zeugopod. In Hoxa11/Hoxd11 double mutants, the ulna and radius are greatly reduced, but not eliminated (Davis et al. 1995). The triple mutants demonstrate a similar extent of reduction, but not complete loss, in the hindlimb zeugopod as the Hoxa11/ Hoxd11 mutant forelimb (D. Wellik and M. Capecchi, unpubl.). In a study by van den Akker et al. (2001), lossof-function of the entire Hox8 paralogous group (Hoxb8, Hoxc8, and Hoxd8) resulted in a variety of transformations of the axial skeleton, but not in loss of these elements. Therefore, that induction of metanephric development is arrested at its initiation with $100 \%$ penetrance would not have been readily predicted.

The present findings leave open the interesting question of what functions other Hox genes are performing during kidney development. The Hox10 paralogous genes are also strongly expressed in the kidney mesenchyme, and mutant combinations of these genes exhibit kidney phenotypes (D. Wellik and M. Capecchi, unpubl.). Misexpression of Hoxd13 in the posterior region of the embryo causes severe kidney defects (Kmita et al. 2000). Determining how these and other Hox genes affect kidney development and their relationship to Hox11 function should provide further insights into the patterning of this model organ.

\section{The role of Hox11 in the pax-eya-six pathway}

The importance of the pax-eya-six transcriptional regulatory hierarchy in organogenesis was first demonstrated in Drosophila eye disc development (Pignoni et al. 1997; Halder et al. 1998; Ohto et al. 1999). In Drosophila, genes homologous to Eya and Six leyes absent [eya] and sine oculis [so], respectively) are downstream of the Pax homolog, eyeless (ey) (Halder et al. 1998). Eya is initially upstream of so, and ectopic expression of eya induces expression of so, but only transiently (Pignoni et al. 1997). Eya and so are both required for induction of ectopic eye formation (Pignoni et al. 1997; Ohto et al. 1999|. Recent studies in vertebrates demonstrated that this pathway is operating in muscle differentiation, mouse lens and placode development as well as in inner ear and metanephric kidney development (Xu et al. 1997, 1999; Heanue et al. 1999).

The present study demonstrates that Hox genes are an important component of the pax-eya-six pathway in mammalian kidney development. In the developing metanephros, the Hox11 paralogs and Eya1 are upstream of Six2. Eya proteins do not contain DNA binding domains, but translocation to the nucleus is important for their function, suggesting that they are likely to be cofactors for DNA binding and transcriptional activation (Ohto et al. 1999). Indeed, previous studies have demonstrated that Eya can bind to Six proteins and can activate downstream genes (for review, see Kawakami et al. 2000). A model consistent with the current study and previous data is that the Hox 11 and Eyal proteins together activate Six2 expression in the metanephric blastema. This activation complex may also include Pax2, as direct interactions between eyeless (the Drosophila Pax homolog) and the sine oculis (so, the Drosophila Six homolog) enhancer region have been reported (Niimi et al. 1999).

With respect to Gdnf regulation, loss-of-function of Pax2, Eya1, or Hox11 paralogs all result in the loss of Gdnf expression (Torres et al. 1995; Xu et al. 1999; this study). Of these, only Pax2 has been shown to bind at the Gdnf locus (Brophy et al. 2001). Pax2 must not be sufficient to activate Gdnf expression, however, because Pax2 expression is normal in both the Eya1 and the Hox11 mutants (Xu et al. 1999; this study). In contrast, Six2 expression is missing in both of these mutants (Xu et al. 1999; this study). Based on the above data and the reported intraregulation within this group, a combination of these proteins including Pax2, Eya1, Six2, and Hox11 could then regulate the expression of GDNF at the appropriate position for metanephric induction and subsequent development.

Although the details of the specific protein interactions occurring in this system remain to be defined, the present findings provide clear insight into the molecular basis of "positional value." This term, along with "positional identity," has been widely used to describe the role of Hox genes in the developing embryo. However, providing a molecular explanation for this observation has been elusive. Hox11 paralogous genes are expressed at the correct time and at the appropriate position along the A-P axis for initiating metanephric kidney formation. By controlling, directly or indirectly, the expression of Gdnf in this region, the Hox genes determine the A-P location for metanephric kidney induction and thus provide this organ with the correct "positional value." Other members of this regulatory pathway, such as Pax2, Eya1, and Six2, are expressed in many regions along the A-P embryonic axis. By coupling with the ap- 
propriate Hox genes to control unique downstream target gene expression, in this case Gdnf, the whole regulatory system acquires the correct positional value.

Consistent with the hypothesis that multiple Hox genes may be used as an integral component of the paxeya-six pathway, Eya1 loss-of-function mutants phenocopy not only the Hox11 kidney phenotype, but many other Hox mutant phenotypes. For example, Eya1 mutants and Hoxa2 mutants exhibit cleft palate (Rijli et al. 1992; Gendron-Maguire et al. 1993; Xu et al. 1999|. Craniofacial defects like those reported for Hoxa3 mutants (Chisaka and Capecchi 1991) and seventh branchiomotor defects similar to Hoxb1 mutants (Goddard et al. 1996) are also reported in Eya1 mutant mice (Xu et al. 1999). Defective cervical vertebrae (i.e., atlas and axis defects) similar to Hoxd3 mutants (Condie and Capecchi 1993) and thoracic vertebral defects similar to $\operatorname{Hox} 7$ and $\operatorname{Hox} 9$ paralogous mutant phenotypes are also observed in Eya1 mutant mice (Chen et al. 1998; Chen and Capecchi 1999; $\mathrm{Xu}$ et al. 1999|. Compellingly, mice with targeted disruption of Eya1, Hoxa1, Pax2, or Gdnf show arrested morphogenesis of the inner ear (Chisaka et al. 1992; Torres et al. 1995; Moore et al. 1996; Pichel et al. 1996; Sanchez et al. 1996; Xu et al. 1999), and mice mutant for Eya1, Hoxa3, or $\operatorname{Pax} 9$ are athymic, aparathyroid, and have reduced thyroid glands (Chisaka and Capecchi 1991; Peters et al. 1998; Xu et al. 1999). These observations support the hypothesis that Hox interaction in the pax-eya-six pathway may be used in multiple contexts during mammalian development.

In summary, we have demonstrated that Hox11 paralogous genes are required for metanephric kidney induction. This function of Hox11 genes was revealed only after the entire paralogous group was disrupted. Control of this inductive process is accomplished at the molecular level by regulating the expression of Six2 and Gdnf, thus revealing a role for the Hox 11 genes in the pax-eyasix transcriptional cascade in metanephric kidney development. The extensive similarities of phenotypes between mice mutant for Eya1, a proposed partner for Six2, and multiple reported Hox loss-of-function mutants suggest that Hox participation in this regulatory pathway may be used in other developmental contexts. Further studies using multiple Hox alleles, molecular analysis, and genetic interaction studies with Eya1 mutant animals will test this hypothesis. Incorporating differentially expressed Hox genes into this regulatory cascade would provide a molecular means of conferring Hox- positional regulatory information to the formation of multiple structures and organs along the A-P axis.

\section{Materials and methods}

\section{PCR genotyping}

Primer sets used to distinguish between wild-type and mutant alleles: Hoxa11, 5' -GCTGGCTTTTATCTGAAGCCGG-3' (forward), 5'-CTCCCAATTCCAGTAGGCTGGA-3' (reverse), 5'-TT GTTCAGACTACAATCTGACC-3' (neo); Hoxc11, 5'-GCAGT TACAGCAGTGGATTTCC-3' (forward), 5'-TGAGCTTTCAG
CGACTGGGTTG-3' (reverse), 5'-CGCGCTCGAGATGTGC TGCAAGGCGATTAA-3' (neo); Hoxd11, 5' -CTTTTTTCCTA TCTCAGTGCCAG-3' (forward), 5'-GGGGTACATCCTGGA GTTCTCA-3' (reverse), 5'-TTCAAGCCCAAGCTTTCGCG AG-3' (neo).

\section{Embryonic histology}

Embryos were dissected in PBS, fixed in Bouin's for less than 24 $\mathrm{h}$ and dehydrated through graded alcohols and stored in $70 \%$ ethanol at $4{ }^{\circ} \mathrm{C}$. Embryonic membranes were used for genotyping. Embryos were vacuum-embedded in paraffin, sectioned at 5 $\mu \mathrm{m}$ and stained with hematoxylin and eosin.

TUNEL assay, immunohistochemistry, and in situ hybridization

TUNEL analysis was performed on paraffin-sectioned embryos using the In Situ Cell Death Detection Kit (Flourescein, Enzo).

For immunochemistry, embryos were fixed in $4 \%$ paraformaldehyde in PBS, processed as paraffin sections, and deparaffinized. Pax2 antibodies (BAbCO, now Covance) were used in a 1:25 to 1:50 dilution on paraffin sections. Wt1 antibodies (Santa Cruz) were used at a 1:4 to 1:5 dilution on paraffin sections.

In situ hybridization procedures followed standard whole mount protocols (Manley and Capecchi 1995; Goddard et al. 1996) through the probe wash. Whole embryos were then washed through a sucrose gradient to $30 \%$, then frozen in OCT and sectioned. Anti-digoxigenin antibody (Boehringer-Mannheim) was added to each PBS-washed slide at a dilution of 1: 2000, and slides were developed in NBT/BCIP. Gdnf in situ hybridization analysis was performed with the addition of three independent digoxigenin-labeled probes, PCR-cloned (TOPO Cloning, Invitrogen) from the 3' untranslated region. The sequences were amplified from reverse-transcribed cDNA, subcloned and labeled by standard methods. Primers used for amplification are as follows:

Set $1,5^{\prime}$-gacttgggtttgggctatga $-3^{\prime}$ and $5^{\prime}$-gcgtcttcaaacacgtgaaa- $3^{\prime}$; Set 2, 5'-tgggctcaacttttgctacc-3' and 5'-cccaagagctagaggctgtg-3'; Set $3,5^{\prime}$-cgagggtccattctcttcag- ${ }^{\prime}$ ' and $5^{\prime}$-'tgggcaaggatagaggaaga- $3^{\prime}$.

At least three to four mutant embryos were used for each experiment.

\section{Acknowledgments}

We acknowledge S.L. Hostikka for the hoxc11 mutant allele (manuscript in preparation). We thank Sheila Barnett, Julie Tomlin, and Jim Hayes for technical support in the IVF procedures. In situ probes for Eya-1 and Six-2 were a gift from Richard Maas.

The publication costs of this article were defrayed in part by payment of page charges. This article must therefore be hereby marked "advertisement" in accordance with 18 USC section 1734 solely to indicate this fact.

\section{References}

Brophy, P.D., Ostrom, L., Lang, K.M., and Dressler, G.R. 2001. Regulation of ureteric bud outgrowth by Pax2-dependent activation of the glial derived neurotrophic factor gene. Development 128: 4747-4756.

Cacalano, G., Farinas, I., Wang, L.-C., Hagler, K., Forgie, A., Moore, M., Armanini, M., Phillips, H., Ryan, A.M., Reichardt, L.F., et al. 1998. GFR $\alpha 1$ is an essential receptor component for GDNF in the developing nervous system and kid- 
ney. Neuron 21: 53-62.

Chen, F. and Capecchi, M.R. 1999. Paralogous mouse Hox genes, Hoxa9, Hoxb9, and Hoxd9, function together to control development of the mammary gland in response to pregnancy. Proc. Nat1. Acad. Sci. 96: 541-546.

Chen, F., Greer, J., and Capecchi, M.R. 1998. Analysis of Hoxa7/ Hoxb7 mutants suggests periodicity in the generation of the different sets of vertebrae. Mech. Dev. 77: 49-57.

Chisaka, O., and Capecchi, M.R. 1991. Regionally restricted developmental defects resulting from targeted disruption of the mouse homeobox gene hox-1.5. Nature 350: 473-479.

Chisaka, O., Musci, T.S., and Capecchi, M.R. 1992. Developmental defects of the ear, cranial nerves and hindbrain resulting from targeted disruption of the mouse homeobox gene Hox-1.6. Nature 355: 516-520.

Condie, B.G. and Capecchi, M.R. 1993. Mice homozygous for a targeted disruption of Hoxd-3 (Hox-4.1) exhibit anterior transformations of the first and second cervical vertebrae, the atlas and the axis. Development 119: 579-595.

- 1994. Mice with targeted disruptions in the paralogous genes hoxa-3 and hoxd-3 reveal synergistic interactions. $\mathrm{Na}$ ture 370: 304-307.

Davis, A.P. and Capecchi, M.R. 1994. Axial homeosis and appendicular skeleton defects in mice with a targeted disruption of hoxd-11. Development 120: 2187-2198.

Davies, J. and Brandli, A. 2002. The Kidney Development Database. http://golgianaedacuk/kidhomehtml.

Davis, A.P., Witte, D.P., Hsieh-Li, H.M., Potter, S.S., and Capecchi, M.R. 1995. Absence of radius and ulna in mice lacking hoxa-11 and hoxd-11. Nature 375: 791-795.

Dressler, G.R. 1999. Kidney development branches out. Dev. Genet. 24: 189-193.

Duboule, D. and Dolle, P. 1989. The structural and functional organization of the murine HOX gene family resembles that of Drosophila homeotic genes. EMBO J. 8: 1497-1505.

Gendron-Maguire, M., Mallo, M., Zhang, M., and Gridley, T. 1993. Hoxa-2 mutant mice exhibit homeotic transformation of skeletal elements derived from cranial neural crest. Cell 75: 1317-1331.

Goddard, J.M., Rossel, M., Manley, N.R., and Capecchi, M.R. 1996. Mice with targeted disruption of Hoxb-1 fail to form the motor nucleus of the VIIth nerve. Development 122: 3217-3228

Graham, A., Papalopulu, N., and Krumlauf, R. 1989. The murine and Drosophila homeobox gene complexes have common features of organization and expression. Cell 57: 367378

Halder, G., Callaerts, P., Flister, S., Walldorf, U., Kloter, U., and Gehring, W.J. 1998. Eyeless initiates the expression of both sine oculis and eyes absent during Drosophila compound eye development. Development 125: 2181-2191.

Heanue, T.A., Reshef, R., Davis, R.J., Mardon, G., Oliver, G., Tomarev, S., Lassar, A.B., and Tabin, C.J. 1999. Synergistic regulation of vertebrate muscle development by Dach2, Eya2, and Six1, homologs of genes required for Drosophila eye formation. Genes \& Dev. 13: 3231-3243.

Hellmich, H.L., Kos, L., Cho, E.S., Mahon, K.A., and Zimmer, A. 1996. Embryonic expression of glial cell-line derived neurotrophic factor (GDNF) suggests multiple developmental roles in neural differentiation and epithelial-mesenchymal interactions. Mech. Dev. 54: 95-105.

Horan, G.S.B., Ramirez-Solis, R., Featherstone, M.S., Wolgemuth, D.J., Bradley, A., and Behringer, R.R. 1995. Compound mutants for the paralogous hoxa-4, hoxb-4, and hoxd-4 genes show more complete homeotic transformations and a dose-dependent increase in the number of vertebrae trans- formed. Genes \& Dev. 9: 1667-1677.

Hostikka, S.L. and Capecchi, M.R. 1998. The mouse Hoxc11 gene: Genomic structure and expression pattern. Mech. Dev. 70: $133-145$

Hsieh-Li, H.M., Witte, D.P., Weinstein, M., Branford, W., Li, H., Small, K., and Potter, S.S. 1995. Hoxa11 structure, extensive antisense transcription and function in male and female fertility. Development 121: 1373-1385.

Kawakami, K., Sato, S., Ozaki, H., and Ikeda, K. 2000. Six family genes-Structure and function as transcription factors and their roles in development. BioEssays 22: 616-626.

Kmita, M., Kondo, T., and Duboule, D. 2000. Targeted inversion of a polar silencer within the HoxD complex re-allocates domains of enhancer sharing. Nat. Genet. 26: 451-457.

Koseki, C., Herzlinger, D., and Al-Awqati, Q. 1992. Apoptosis in metanephric development. J. Cell Biol. 119: 1327-1333.

Kreidberg, J.A., Sariola, H., Loring, J.M., Maeda, M., Pelletier, J., Housman, D., and Jaenisch, R. 1993. WT-1 is required for early kidney development. Cell 74: 679-691.

Kuure, S., Vuolteenaho, R., and Vainio, S. 2000. Kidney morphogenesis: Cellular and molecular regulation. Mech. Dev. 92: $31-45$.

Manley, N.R. and Capecchi, M.R. 1995. The role of Hoxa-3 in mouse thymus and thyroid development. Development 121: 1989-2003.

Moore, M.W., Klein, R.D., Farinas, I., Sauer, H., Armanini, M., Phillips, H., Reichardt, L.F., Ryan, A.M., Carver-Moore, K., and Rosenthal, A. 1996. Renal and neuronal abnormalities in mice lacking GDNF. Nature 382: 76-79.

Niimi, T., Seimiya, M., Kloter, U., Flister, S., and Gehring, W.J. 1999. Direct regulatory interaction of the eyeless protein with an eye-specific enhancer in the sine oculis gene during eye induction in Drosophila. Development 126: 2253-2260.

Ohto, H., Kamada, S., Tago, K., Tominaga, S.-I., Ozaki, H., Sato, S., and Kawakami, K. 1999. Cooperation of Six and Eya in activation of their target genes through nuclear translocation of Eya. Mol. Cell. Biol. 19: 6815-6824.

Patterson, L.T., Pembaur, M., and Potter, S.S. 2001. Hoxa11 and Hoxd11 regulate branching morphogenesis of the ureteric bud in the developing kidney. Development 128: 2153-2161.

Peters, H., Neubuser, A., Kratochwil, K., and Balling, R. 1998. Pax9-deficient mice lack pharyngeal pouch derivatives and teeth and exhibit craniofacial and limb abnormalities. Genes \& Dev. 12: 2735-2747.

Pichel, J.G., Shen, L., Sheng, H.Z., Granholm, A.-C., Drago, J., Grinberg, A., Lee, E.J., Huang, S.P., Saarma, M., Hoffer, B.J., et al. 1996. Defects in enteric innervation and kidney development in mice lacking GDNF. Nature 382: 73-76.

Pignoni, F., Hu, B., Zavitz, K.H., Xiao, J., Garrity, P.A., and Zipursky, S.L. 1997. The eye-specification proteins So and Eya form a complex and regulate multiple steps in Drosophila eye development. Cell 91: 881-891.

Rijli, F.M., Mark, M., Lakkaraju, S., Dierich, A., Dolle, P., and Chambon, P. 1993. A homeotic transformation is generated in the rostral branchial region of the head by disruption of Hoxa-2, which acts as a selector gene. Cell 75: 1333-1349.

Rossel, M. and Capecchi, M.R. 1999. Mice mutant for both Hoxa1 and Hoxb1 show extensive remodeling of the hindbrain and defects in craniofacial development. Development 126: 5027-5040.

Sainio, K., Suvanto, P., Davies, J., Wariovaara, J., Wartiovaara, K., Saarma, M., Arumae, U., Meng, X., Lindahl, M., Pachnis, V., et al. 1997. Glial-cell-derived neurotrophic factor is required for bud initiation from ureteric epithelium. Development 124: 4077-4087.

Sanchez, M.P., Silos-Santiago, I., Frisen, J., He, B., Lira, S.A., and 
Wellik et al.

Barbacid, M. 1996. Renal agenesis and the absence of enteric neurons in mice lacking GDNF. Nature 382: 70-73.

Schuchardt, A., D'Agati, V., Pachnis, V., and Costantini, F. 1996. Renal agenesis and hypodysplasia in ret-k- mutant mice result from defects in ureteric bud development. Development 122: 1919-1929.

Small, K.M. and Potter, S.S. 1993. Homeotic transformations and limb defects in Hox A11 mutant mice. Genes \& Dev. 7: 2318-2328.

Studer, M., Gavales, A., Marshall, H., Ariza-McNaughton, L., Rijli, F.M., Chambon, P., and Krumlauf, R. 1998. Genetic interactions between Hoxa1 and Hoxb1 reveal new roles in regulation of early hindbrain patterning. Development 125: 1025-1036.

Sztein, J., Farley, J., Young, A., and Mobraaten, L. 1997. Motility of cryopreserved spermatazoa affected by temperature of collection and rate of thawing. Cryobiology 35: 46-52.

Torres, M., Gomez-Pardo, E., Dressler, G.R., and Gruss, P. 1995. Pax-2 controls multiple steps of urogenital development. Development 121: 4057-4065.

Treanor, J.J.S., Goodman, L., Sauvage, F.D., Stone, D.M., Poulsen, K.T., Beck, C.D., Gray, C., Armanini, M.P., Pollock, R.A., Hefti, F., et al. 1996. Characterization of a multicomponent receptor for GDNF. Nature 382: 80-83.

Trupp, M., Arenas, E., Fainzilber, M., Nilsson, A.-S., Sieber, B.-A., Grigoriou, M., Kilkenny, C., Salazar-Grueso, E., Pachnis, V., Arumae, U., et al. 1996. Functional receptor for GDNF encoded by the c-ret proto-oncogene. Nature 381: 785-789.

van den Akker, E., Fromental-Ramain, C., de Graaff, W., Mouellic, H.L., Brulet, P., Chambon, P., and Deschamps, J. 2001. Axial skeletal patterning in mice lacking all paralogous group 9 Hox genes. Development 128: 1911-1921.

Xu, P.-X., Woo, I., Her, H., Beier, D.R., and Maas, R.L. 1997. Mouse Eya homologs of the Drosophila eyes absent gene require Pax6 for expression in lens and nasal placode. Development 124: 219-231.

Xu, P.-X., Adams, J., Peters, H., Brown, M.C., Heaney, S., and Maas, R. 1999. Eya-1-deficient mice lack ears and kidneys and show abnormal apoptosis of organ primordia. Nat. Genet. 23: 113-117.

Zakany, J., Fromental-Ramain, C., Warot, X., and Duboule, D. 1997. Regulation of number and size of digits by posterior Hox genes: A dose-dependent mechanism with potential evolutionary implications. Proc. Natl. Acad. Sci. 94: 1369513700. 


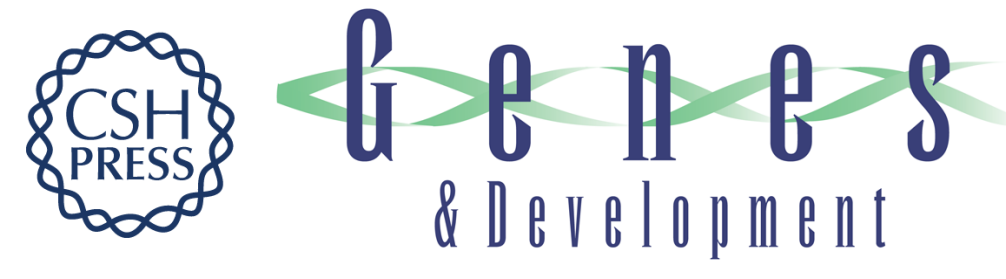

\section{Hox11 paralogous genes are essential for metanephric kidney induction}

Deneen M. Wellik, Patrick J. Hawkes and Mario R. Capecchi

Genes Dev. 2002, 16:

Access the most recent version at doi:10.1101/gad.993302

References This article cites 49 articles, 24 of which can be accessed free at: http://genesdev.cshlp.org/content/16/11/1423.full.html\#ref-list-1

License

Email Alerting

Receive free email alerts when new articles cite this article - sign up in the box at the top Service right corner of the article or click here.

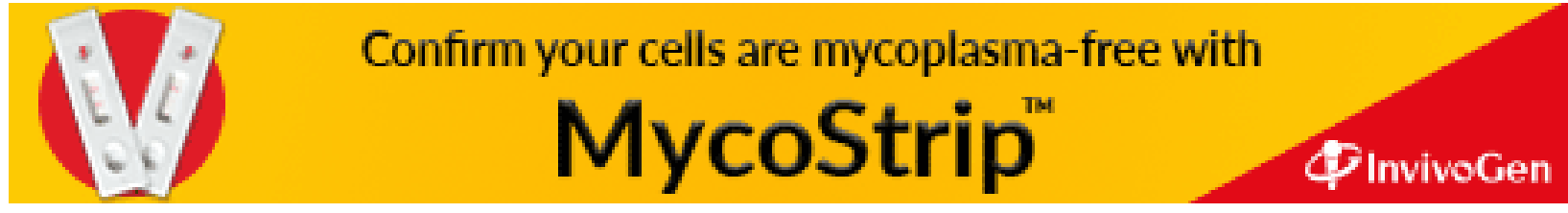

